\title{
Target Tracking and Obstacle Avoidance for Mobile Robot based on Kinect
}

\author{
Mengxin $\mathrm{LI}^{1,}$ a ,Jiadi $\mathrm{YIN}^{2, \mathrm{~b}}$ \\ ${ }^{1}$ School of Information and Control Engineering Shenyang Jianzhu University Shenyang,110179 \\ China \\ ${ }^{2}$ School of Information and Control Engineering Shenyang Jianzhu University Shenyang, 110179 \\ China \\ a limengxin@sjzu.edu.cn b1304007591@qq.com
}

Keywords:mobile robot;Target tracking ;obstacle avoidance;Kinect;BFO-PF;artificial potential field;

\begin{abstract}
In order to get a better understanding of the obstacle avoidance of a mobile robot when it is tracking a target,we used Kinect to take the place of the traditional range radar and camera.Tracking part uses the improved particle filter algorithm. To deal with the particle degeneracy problem, a new particle filter (PF) based on the Bacteria Foraging Optimization (BFO) algorithm is proposed.Improved artificial potential field method increases the safe area which was applied to remove the information of an obstacle not disturbing the motion of the robot,so as to improve the ability of the mobile robot to pass through a narrow passage by the traditional artificial potential field method.The results show that the system can accomplish the task of tracking and obstacle avoidance.
\end{abstract}

\section{Introduction}

Ultrasonic and laser ranging radar is the main sensor of the mobile robot to obtain environmental information. However, the ultrasonic radar to obtain the environmental information is more rough, laser radar, although the acquisition of information is rich, but the cost is very high. Kinect is a 3-D camera produced by the Microsoft Corp. It can obtain the position information of the environment optical image and optical image. The information is rich and low cost. It is suitable for mobile robot path planning and target tracking.

In this paper, the target tracking and obstacle avoidance system of mobile robot is constructed by using Kinect instead of traditional ranging sensor.Combined with the improved artificial potential field obstacle avoidance algorithm[1-4] and particle filer (PF) based on the Bacteria Foraging Optimization (BFO) algorithm[5-7], the target tracking is realized on the basis of the differential driven wheeled mobile robot.

\section{Mobile Robot With Kinect}

\section{Mobile Robot Kinematic Model}

At present, the mobile robot is used for the wheel differential drive, which is described in the inertial coordinate system

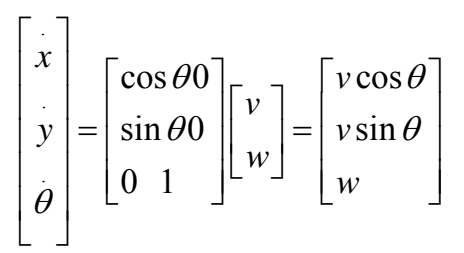

where: $x, y$ coordinates of the mobile robot in the global reference frame, $\theta$ is the direction of the mobile robot in the global reference frame angle, $v$ is the speed of the mobile robot moves linearly, 
$\omega$ is the angular velocity of rotation of the mobile robot. Figure 1 is a mobile robot inertial coordinate system, where $\mathrm{Xr}, \mathrm{Yr}$ determine the local coordinate system of the mobile robot.X,Y established its world coordinates.

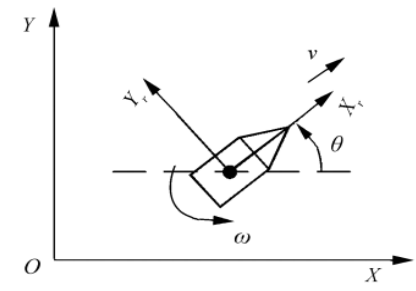

Fig.1 Inertial coordinate system of the mobile robot

According to correspondence between the mobile robot global coordinate system and local coordinate system, the local coordinate system can transform coordinates relative to the global coordinate system of coordinates. Order the coordinates $\left(x_{l}, y_{l}\right)$ to coordinate robot local coordinate system, and it is known in the global mobile robot coordinate system position $\left(x_{c}, y_{c}, \theta_{c}\right)$, then the relative coordinates global robot coordinate system $\left(x_{g}, y_{g}\right)$ by equation (1).

$$
\left\{\begin{array}{l}
x_{g}=x_{c}+x_{l} \cos \theta_{c}-y_{l} \sin \theta_{c}, \\
y_{g}=y_{c}+x_{l} \sin \theta_{c}+y_{l} \cos \theta_{c} .
\end{array}\right.
$$

Kinect

Microsoft launched Kinect somatosensory camera one for XBOX360, currently supports the Kinect software development kit for the use of personal computers and Microsoft have OpenNI provided SDK, Kinect via USB Interface connected to the computer. Use Kinect can get a resolution RGB color images was $640^{*} 480$ and a corresponding depth image. The use of the depth information obtained, can be obtained by converting the corresponding 3- D model of the environment, the converted three-dimensional coordinate system shown in Figure 2, the origin $\mathrm{O}$ of the camera location, $\mathrm{Z}$-axis positive direction in front of the camera.
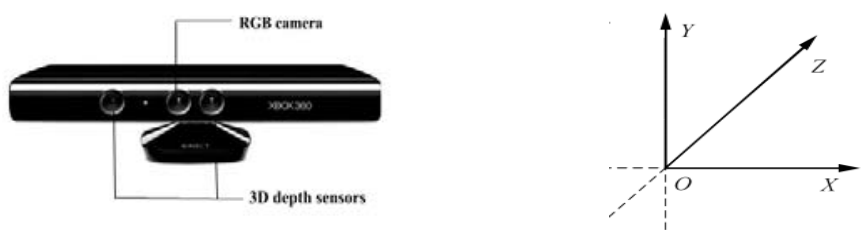

Fig.2 Kinect and image coordinate system

Since different coordinate systems coordinate system Kinect camera and mobile robots, so 3- D coordinate obtained from Kinect need to go through the conversion in order to ensure consistency with the mobile robot coordinate system. Specific conversion relationship shown in Fig.3, the figure is the Kinect for 3- D X,Y,Z coordinate system, $\mathrm{Xr}, \mathrm{Yr}$ is local coordinate system for the mobile robot.

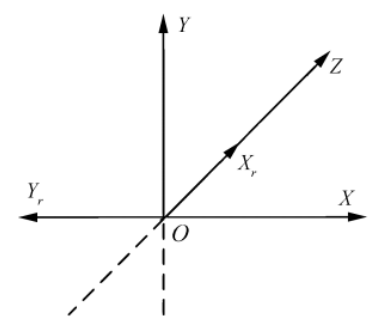

Fig.3 Coordinates relationship between Kinect and mobile robot

\section{Target Tracking and Obstacle Avoidance}

Particle Filter Algorithm Based On Bacterial Foraging Optimization

1) The core of the bacterial foraging algorithm is the trend of the inner layer. Generally, trend 
operation is just a random search method.The method avoids the blindness of random search and improves the searching efficiency. It is given by the following.

$$
\begin{aligned}
& \phi(j)=\operatorname{sign}\left(\theta^{\text {best }}-\theta^{i}\right) \\
& \theta^{i}(j+1, k, l)=\theta^{i}(j, k, l)+(C(i) \llbracket \operatorname{rand}()) \phi(j)
\end{aligned}
$$

The proposed method (3) gives the improved search direction, which $\theta^{\text {best }}$ represents the position of the current group of individuals, and sign (•)represents the symbolic function; (4) gives the final trend operating formula, which rand() means that a random number $U[0,1]$ of the distribution, the introduction of random step size factor can expand the search area and avoid the condition that the fixed step size is likely to miss the optimal search results.

2) Migration operation is the trend of the operation, its essence is the migration of individuals with a certain probability. chromosome mutation, which is similar to the genetic algorithm in the literature therefore, mutation method proposed by [8] for the basic migration operation is improved.

$$
\theta^{i}(j, k, l+1)=\theta^{i}(j, k, l)+c
$$

In the upper formula, $\mathrm{c}$ is a Gauss random variable, $c \sim N\left(y(t)-\theta^{i}, q\right)$ is subject to the

3)Simulation And Analysis observation of the current time, and $q$ is defined as a constant of the variance.

According to the literature $[9,10,11]$ state equation and observation equation using non-stationary growth model, the model by as follows.

$$
\left\{\begin{array}{l}
x_{k}=0.5 x_{k-1}+\frac{25 x_{k-1}}{1+x_{k-1}^{2}}+8 \cos [1.2(k-1)]+u_{k-1} \\
y_{k}=\frac{x_{k}^{2}}{20}+v_{k}
\end{array}\right.
$$

The above model,state noise $u_{k} \sim N(0,10)$, observation noise $v_{k} \sim N(0,0.1)$, both are independent. The initial state of the system is set to 0.1. Respectively with SPF, GA-PF, APF, and BFO-PF algorithm the model proposed state estimation.

Bacterial foraging algorithm parameters set, $N_{c}=4, N_{r}=2 N_{e d}=2, P_{e d}=0.8$,

Genetic parameter is set, $N=20, P_{\text {cross }}=0.6 P_{\text {mutation }}=0.4$,

The number of particles is set to 100, Fig. 4shows the simulation results of a single experiment.

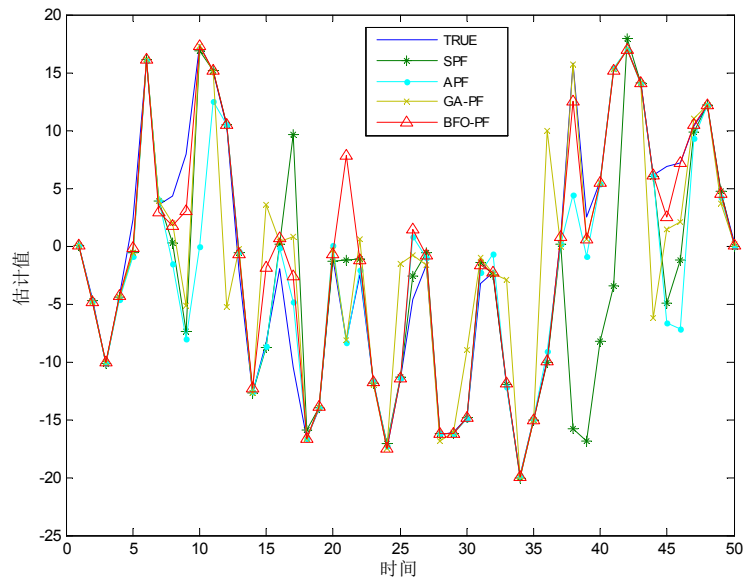

Fig. 4 Tracking performance of different algorithms

As it can be seen from Fig. 4, compared with other algorithms, BFO-PF algorithm in real value tracking process, closer to the real value. To further verify the performance of the algorithm, using the root mean square error of several Monte Carlo experiments (RMSE) as an evaluation index, root mean square error is defined multiple Monte Carlo experiment is given by the following equation.

$$
\text { RMSE }=\sqrt{\frac{1}{M C} \sum_{k=1}^{M C}\left(\boldsymbol{x}_{k}-\boldsymbol{x}_{k}\right)^{2}}
$$

where, MC for the Monte Carlo trials, $x_{k}$ is the state of true value, $x_{k}$ the state estimated value.

Particle number is set to 100 , the monte carlo experiments for 400. Table 1 shows the RMSE of 
mean and variance of different algorithm, and the average elapsed time.

TABLE I. RMSE mean and variance of different algorithms and the average running time

\begin{tabular}{lccc}
\hline $\begin{array}{c}\text { Particle } \\
\text { filtering } \\
\text { method }\end{array}$ & $\begin{array}{c}\text { RMSE } \\
\text { Mean }\end{array}$ & $\begin{array}{c}\text { RMSE } \\
\text { Variance }\end{array}$ & $\begin{array}{c}\text { Average } \\
\text { running } \\
\text { time }\end{array}$ \\
\hline SPF & 5.7557 & 1.3490 & 0.0658 \\
APF & 6.9394 & 1.5339 & 0.1296 \\
GA-PF & 5.6305 & 1.2792 & 0.6354 \\
BFO-PF & 5.4030 & 1.2646 & 0.5980 \\
\hline
\end{tabular}

From table 1 , it can be seen that the BFO-PF method proposed in this paper is superior to APF and SPF algorithm in filtering performance.It shows the efficiency of the algorithm.

Obstacle Avoidance

1)Security zone artificial potential field

Obstacle avoidance of mobile robot using artificial potential field is completed, the method is simple and practical and clear physical significance and thus subject to extensive research and use. Specific formula for artificial potential field.In order to achieve the goal of target tracking and obstacle avoidance between switching design with regional security of the artificial potential field method, implementation methods, such as Fig.5. Fig.5(a) a solid rectangle frame representation of the normal running of regional security, Fig.5(b) a solid rectangle box have obstacle 3, at this point the system preferentially into the obstacle avoidance mode, mobile robot according to the dotted rectangle frame determined within the scope of the obstacle information obstacle avoidance until the small box there is no obstacle. This method can not only effectively control mode switching, but also enhances the whole system through the narrow channel and the ability to walk along the wall.

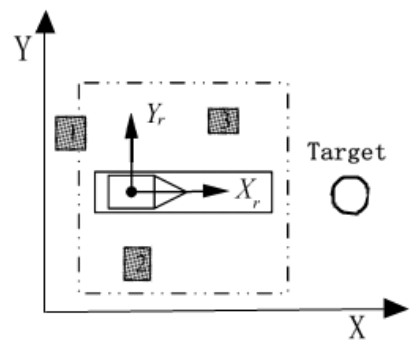

(a)No obstacle

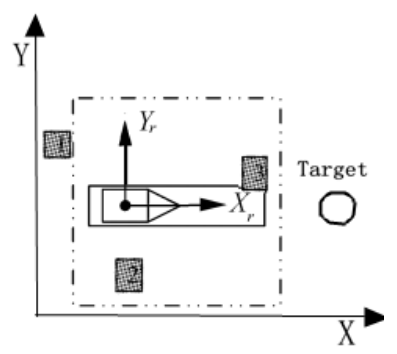

(b)obstacle

Fig. 5 The safe region of artificial potential

2)Experimental results and analysis

The experiment used a length of $900 \mathrm{~mm}$ width is about $660 \mathrm{~mm}$ height $480 \mathrm{~mm}$ mobile robot using a differential drive mode, and no turning radius limit. Inside this robot was equipped with a computer, you can easily connect with Kinect.The experimental results shown in Fig.6, shown in red dots indicate the mobile robot trajectory, long black bars represent obstacles, rectangle shows a mobile robot. Mobile robot eventually stops moving target at a distance of about d, set D in the experiment is $1 \mathrm{~m}$. As can be seen from Fig. 6 mobile robots in tracking targets while moving to effectively avoid obstacles.

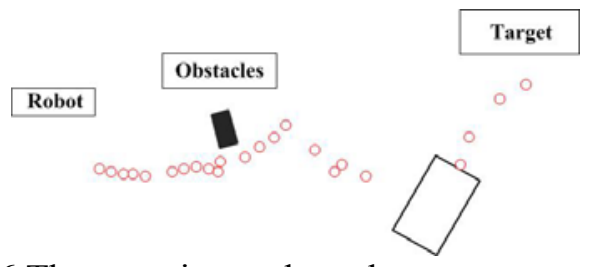

Fig.6 The experimental results 


\section{Conclusion}

Experimental results show that the use of Kinect may well complete mobile robot target tracking and obstacle avoidance task, we propose a bacterial foraging optimization based on particle filter resampling improved particle depletion problem exists particle filter. In this paper, two-dimensional model, in future studies, if the three-dimensional model into the algorithm, mobile robot will be able to better track and obstacle avoidance.

\section{Acknowledgement}

In this paper, the research was sponsored by the Nature Science Foundation of China (Project No. 61440004)

\section{References}

[1]GAO Feng, WANG Jiangfeng, SHI Shaoyou, et al. Vehicle intelligent collision warning algorithm based on fuzzy neural networks $[\mathrm{J}]$. Journal of Jiangsu University: Nature Science Edition, 2006, 27( 3): 211-215.

[2]WANG Wei, WANG Hua, School of Astronautics. Real-time obstacle avoidance trajectory planning for missile borne air vehicle based on constrained artificial potential field method[J]. Journal of Aerospace Power, 2014.

[3]Liu Z Z. The Application of Artificial Potential Field in Intelligent Unmanned Vehicle Obstacle Avoidance System[J]. Computing Technology \& Automation, 2013.

[4]Yang Y B, Wang C L. Obstacle Avoidance Method for Mobile Robots Based on Improved Artificial Potential Field Method and Its Implementation on MATLAB[J]. Journal of University of Shanghai for Science \& Technology, 2013.

[5]YangXin, LiuJia, Pengyu Zhou, etc. Based on feature fusion of adaptive particle filter target tracking algorithm . Journal of Jilin university (engineering science), 2015, 2:029.

[6]Ruiji Zhu , Naixin Qi, shengxiu Zhang, et al. A kind of improved infrared target tracking algorithm based on particle filter . Journal of Sichuan armaments factories, 2014, 35 (3) : 133-136.

[7]XIA Xuan, LIU Huaping. DSP- based active vision system[J]. Robot, 2012, 34( 3): 354- 362.

[8]PARK S., WANG J.H., ROU K., KIM E. A New Particle Filter Inspired by Biological Evolution: Genetic Filter. World Academy of Science, Engineering and Technology 33, 2007

[9] Lei Liang, Lili Zhao. Based on artificial fish swarm algorithm (J). The computer is applied to software, 2012,29 (1): 137-140.

[10] Zhiyu Zhu. Particle filter algorithm and its application. Science Press, 2010: 74 -97

[11]ARULAMPALAM M. S., MASKELl S., GORDON N., CLAPP T. A Tutorial on Particle Filters for Online Nonliner/Non-Gaussian Bayesian Tracking. IEEE Trans. on Signal Processing, Vol.50 No.2 February 2002. 